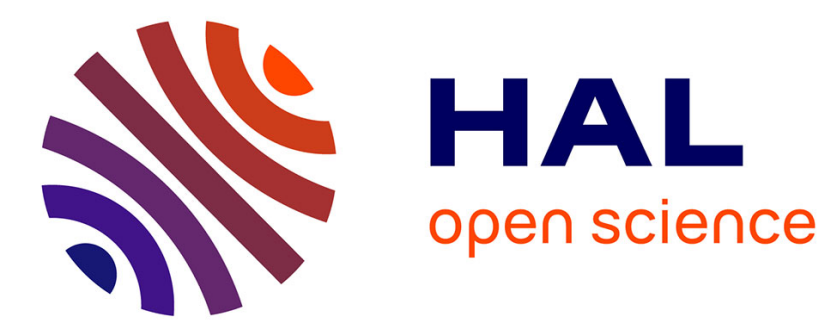

\title{
Generic Solving of One-compartment Toxicokinetic Models
}

\author{
Sandrine Charles, Aude Ratier, Christelle Lopes
}

\section{To cite this version:}

Sandrine Charles, Aude Ratier, Christelle Lopes. Generic Solving of One-compartment Toxicokinetic Models. Journal of Exploratory Research in Pharmacology, 2021, 10.14218/JERP.2021.00024 . hal03362341

\section{HAL Id: hal-03362341 \\ https://hal.science/hal-03362341}

Submitted on 1 Oct 2021

HAL is a multi-disciplinary open access archive for the deposit and dissemination of scientific research documents, whether they are published or not. The documents may come from teaching and research institutions in France or abroad, or from public or private research centers.
L'archive ouverte pluridisciplinaire HAL, est destinée au dépôt et à la diffusion de documents scientifiques de niveau recherche, publiés ou non, émanant des établissements d'enseignement et de recherche français ou étrangers, des laboratoires publics ou privés.

\section{다)(1) $(5$}

Distributed under a Creative Commons Attribution - NonCommerciall 4.0 International 


\title{
Generic Solving of One-compartment Toxicokinetic Models
}

\author{
Sandrine Charles $\#^{*} \oplus$, Aude Ratier ${ }^{\#}$ and Christelle Lopes \\ Biometry and Evolutionary Biology Laboratory, University of Lyon, University Lyon 1, UMR CNRS 5558, Villeurbanne, France
}

Received: July 15, 2021 | Revised: August 15, 2021 | Accepted: August 24, 2021 | Published: Month 00, 2021

\begin{abstract}
This paper gives the full analytical solution of the generic set of ordinary differential equations that define onecompartment toxicokinetic models. These models describe the uptake and elimination processes that occur within living organisms when exposed to chemical substances. The models solved in this paper consider living organisms as a unique compartment, into which a parent compound enters via several possible exposure routes and from which it is eliminated as well as its potential metabolites. Benefiting from generic solutions of onecompartment toxicokinetic models is particularly useful when fitting them to experimental data, facilitating the writing of the inference algorithms leading to parameter estimates. Additionally, these models are of crucial interest in environmental risk assessment for the calculation of bioaccumulation metrics as required by regulators in support of decision-making when they evaluate dossiers for marketing authorisation of active substances.
\end{abstract}

\section{Introduction}

In this paper, we consider a generic one-compartment toxicokinetic (TK) model describing uptake and elimination processes within living organisms when exposed to chemical substances. From a general point-of-view, the term TK refers to the movement and fate (also called disposition) of chemical substances that are potentially toxic. In particular, this term is used when describing the time course of absorption, distribution and elimination (accounting for both biotransformation and excretion) of substances within organisms (ADME). Toxicokinetics is also related to pharmacokinetics and is often considered as the same scientific field, the main difference being linked to the type of compound (toxicants or pharmaceuticals). In comparison with pharmaceutical exposure, exposure to toxicants is often uncontrolled and variable over time predominantly at larger concentrations. ${ }^{1}$

Mathematical TK models are particularly useful to predict the

Keywords: Ordinary differential equations; Environmental risk assessment; Living organisms; Active substances; Toxicokinetic models; Bioaccumulation metrics. Abbreviations: ADME, absorption, distribution, metabolism and excretion; EFSA, European food safety authority; ERA, environmental risk assessment; ODE, ordinary differential equation; PBPK, physiologically based pharmacokinetic; PBTK, physiologically based toxicokinetic; TK, toxicokinetic.

${ }^{*}$ Correspondence to: Sandrine Charles, Biometry and Evolutionary Biology Laboratory, University of Lyon, University Lyon 1, UMR CNRS 5558, Villeurbanne 69100 France. ORCID: https://orcid.org/0000-0003-4604-0166. Tel: +33 (0)4 7243 2900, E-mail: sandrine.charles@univ-lyon1.fr

\#Both authors contributed equally to this work.

How to cite this article: Charles S, Ratier A, Lopes C. Generic Solving of Onecompartment Toxicokinetic Models. J Explor Res Pharmacol 2021;00(00):00-00. doi: 10.14218/JERP.2021.00024. adverse effects of xenobiotics or to prevent undesired residues within animal tissues from entering the human food chain. ${ }^{2}$ Indeed, TK models allow the prediction of tissue concentrations over time, involving several kinetic parameters, such as uptake and elimination rates, from which useful metrics for assessing risk may be derived. ${ }^{3}$ Additionally, TK models can be used to facilitate a better understanding of the underlying physiological mechanisms driving chemical ADME processes. ${ }^{4,5}$

A wide variety of TK models exists, chosen from both the available data and the use intended for the model (e.g., describe and/ or predict); simple or more complex models may be preferred accordingly. ${ }^{6}$ Basically, we distinguish classic TK models from physiologically-based (PB) TK models, referred to as PBTK, or PBPK (standing for PB Pharmaco-Kinetic) models in the field of pharmacology. As fully detailed in Gehring et al., ${ }^{7}$ PBPK models are a crucial tool in Environmental Risk Assessment (ERA) for regulatory bodies, such as the US Environmental Protection Agency (US EPA) and European Food Safety Authority (EFSA). ${ }^{8,9}$ In fact, PBPK models are an ethical and scientifically sound method to predict exposure to toxic xenobiotics in humans through animalto-human extrapolation or based on human biomonitoring data. (PB)TK models are also of particular interest for ERA, when the calculation of bioaccumulation metrics is required by regulators to evaluate dossiers for substance marketing authorizations. ${ }^{10-12}$

The generic TK model we unravel in this paper is a one-compartment model that considers organisms as a whole, in which chemical compounds may enter and from which these compounds can be eliminated. More complex TK models (namely PBTK models) refine the description of contamination pathways within organisms, distinguishing organs and tissues from physiological hypotheses on potential targets of exposure compounds; PBTK models have been 
Table 1. Symbols, meaning, and units of all parameters and variables involved in the full set of ordinary differential equations defining the generic onecompartment toxicokinetic model

\begin{tabular}{|c|c|}
\hline Symbol & Meaning and unit \\
\hline$t$ & time (in time units) \\
\hline$t_{c}$ & duration of the accumulation phase (in time units) \\
\hline$C_{p}(t)$ & internal concentration of the parent compound at $t$ (in $\mu g \cdot g^{-1}$ ) \\
\hline$C_{m_{\ell}}(t)$ & internal concentration of metabolite $\ell$ at $t$ (in $\mu g \cdot g^{-1}$ ) \\
\hline$U=\sum_{i=1}^{I} k_{u_{i}} c_{i}$ & sum of all uptake terms \\
\hline$c_{i}$ & concentration via the exposure route $i$ (in $\mu g \cdot m L^{-1}$ ) \\
\hline$E=\sum_{j=1}^{J} k_{e_{j}}$ & sum of all elimination terms for the parent compound \\
\hline$M=\sum_{\ell=1}^{L} k_{m_{\ell}}$ & sum of all elimination terms for metabolite $\ell$ \\
\hline$i$ & index of exposure sources, $i=1 . . . I$ \\
\hline$j$ & index of elimination processes, $j=1 \ldots J$ \\
\hline e & index of metabolites, $\ell=1 \ldots L$ \\
\hline I & total number of exposure sources (\#) \\
\hline J & total number of elimination processes (\#) \\
\hline$L$ & total number of metabolites (\#) \\
\hline$k_{u_{i}}$ & uptake rate of exposure source $i$ (per time units) \\
\hline$k_{e_{j}}$ & excretion rates of elimination process $j$ (per time units) \\
\hline$k_{e_{\ell}}$ & excretion rates of metabolite $\ell$ (per time units) \\
\hline$k_{m_{\ell}}$ & metabolization rate of metabolite $\ell$ (per time units) \\
\hline$R=\frac{U}{E+M}$ & NA \\
\hline$D_{\ell}=k_{e_{\ell}}-(E+M)$ & $N A, \forall \ell=1 \ldots L$ \\
\hline$Q=C_{0}-R\left(1-e^{(E+M) t_{c}}\right)$ & NA \\
\hline
\end{tabular}

\# stands for numbers, while NA means 'Not Applicable'

mainly developed for humans ${ }^{13,14}$ and big mammals. ${ }^{15,16}$ Recently, a PBTK model was proposed by Gestin et $a l .{ }^{17}$ for invertebrates, pushing the immense potential of these models a step further.

Our TK model, even if not fully refined as a PBTK model, has the great advantage of accounting for several exposure sources (e.g., water, sediment, and/or food), several elimination processes (e.g., direct elimination, dilution by growth and/or biotransformation), and several potential metabolites of the parent chemical compound to which organisms are exposed. ${ }^{18}$ The next section details the dynamic system of ordinary differential equations (ODEs) and the mathematical exact solution, followed by a summary of the full set of ODE in section 3. Then, section 4 illustrates the use of model based on simulations from parameter values obtained by the study of real datasets.

\section{One-compartment TK set of ODE}

Our generic one-compartment TK model is composed of two sets of ODE: accumulation and depuration sets. The accumulation phase includes both absorption and elimination processes, as those related to the biotransformation of the parent compound into metabolites, during which organisms are exposed to a given compound. The depuration phase concerns only elimination processes, including biotransformation, during which organisms are transferred into a clean medium. The transition from one set of ODE to the other takes place at time $t_{c}$ corresponding to the duration of the accumulation phase (see Table 1).

In practice, the exposure concentration to which organisms are exposed may vary over time in real environments. This means 
there is no analytical solution of the TK model, so that only a numerical solution can be obtained with an appropriate algorithm. Therefore, our work assumes that the exposure concentration remains constant over time regardless of the exposure source. Such an experimental condition can be ensured for most chemical compounds when performing laboratory experiments. In addition, this assumption provides the exact solution of the full one-compartment TK model by considering as many routes of exposure and as many elimination processes as desired, as well as an infinite number of phase I metabolites, i.e., directly derived from the parent compound to which organisms are exposed. Kuo et al. ${ }^{19}$ previously suggested a partially resolved one-compartment TK model but only for the accumulation phase and one exposure route. In a similar manner, D'Argenio et al. ${ }^{12}$ published an analytical solution for a linear multi-compartments TK model with a non-zero initial condition.

\section{Accumulation phase $\left(0 \leq t \leq t_{c}\right)$}

The set of ODEs describing the accumulation phase $\left(0 \leq t \leq t_{c}\right)$ is as follows:

$$
\left\{\begin{array}{l}
\frac{d C_{p}(t)}{d t}=U-(E+M) \times C_{p}(t) \\
\frac{d C_{m \ell}(t)}{d t}=k_{m \ell} \times C_{p}(t)-k_{e \ell} \times C_{m \ell}(t), \forall \ell=1 \cdots L \text { (b) }
\end{array}\right.
$$

All parameters and variables, with their meaning and units when applicable, are provided together in Table 1.

Equation (1.a) for the parent compound is a linear first-order ODE with constant coefficients and a second member. Equation (1.a) admits $C_{p a r}(t)=\frac{U}{E+M}=R$ as a particular solution. Equation (1.a) without its second member is:

$\frac{d C_{p}(t)}{d t}-(E+M) \times C_{p}(t)=0 \Leftrightarrow C_{p}(t)=K \times e^{-(E+M) \times t}, K \in \mathbb{R}^{+}$

Given the initial condition $C(t=0)=C_{0}\left(C_{0} \geq 0\right)$, we can obtain the full analytical solution of Equation (1.a), providing that the internal concentration of the parent compound over time during the accumulation phase $\left(0 \leq t \leq t_{c}\right)$ is as follows:

$$
C_{p}(t)=\left(C_{0}-R\right) \times e^{-(E+M) \times t}+R
$$

See Table 1 for the definition of parameter $R$.

Equation (1.b) is also a linear first-order ODE with constant coefficients and a second member, with the following analytical solution when the second member is removed:

$$
\frac{d C_{m_{\ell}}(t)}{d t}-k_{e_{\ell}} \times C_{m_{\ell}}(t)=0 \Leftrightarrow C_{m_{\ell}}(t)=K \times e^{-k_{e_{\ell}} \times t}, K \in \mathbb{R}^{+}, \forall \ell=1 \ldots L
$$

The method of variation of a constant consists of writing the general solution of Equation (1.b) as:

$$
C_{m_{\ell}}(t)=K(t) \times e^{-k_{e_{\ell}} \times t}
$$

which is used to find function $K(t)$ by deriving $C_{m_{\ell}}(t)$ then insert the result into Equation (5). The derivative of Equation (1.b) then becomes:

$$
\frac{d C_{m_{\ell}}(t)}{d t}=\frac{d K(t)}{d t} \times e^{-k_{e_{\ell}} \times t}-K(t) \times k_{e_{\ell}} \times e^{-k_{e_{\ell}} \times t}
$$

Inserting the result from the previous equation into Equation (1.b) leads to:

$$
\frac{d K(t)}{d t}=k_{m_{\ell}} \times\left(\left(C_{0}-R\right) \times e^{D_{\ell} \times t}+R \times e^{k_{e_{\ell}} \times t}\right)
$$

which integrates into:

$$
K(t)=k_{m_{\ell}} \times\left(\frac{C_{0}-R}{D_{\ell}} \times e^{D_{\ell} \times t}+\frac{R}{k_{e_{\ell}}} \times e^{k_{e_{\ell}} \times t}\right)+C, C \in \mathbb{R}
$$

See Table 1 for the definition of parameter $D_{\ell}$.

The general solution of Equation (1.b) finally becomes:

$$
C_{m_{\ell}}(t)=k_{m_{\ell}} \times\left(\frac{C_{0}-R}{D_{\ell}} \times e^{-(E+M) \times t}+\frac{R}{k_{e_{\ell}}}\right)+C \times e^{-k_{e_{\ell}} \times t}, C \in \mathbb{R}
$$

When considering the initial condition $C_{m_{\ell}}(t=0)=0$ when the accumulation phase starts, organisms are only exposed to the parent compound, so that there are no metabolites. With this condition, we can finally acquire the full analytical solution of Equation (1.b), providing the internal concentration of metabolite $\ell$ over time during the accumulation phase:

$$
\begin{gathered}
C_{m_{\ell}}(t)=k_{m_{\ell}} \times\left\{\frac{C_{0}-R}{D_{\ell}} \times\left(e^{-(E+M) \times t}-e^{-k_{e_{\ell}} \times t}\right)\right. \\
\left.+\frac{R}{k_{e_{\ell}}} \times\left(1-e^{-k_{e_{\ell}} \times t}\right)\right\}
\end{gathered}
$$

\section{Depuration phase $(t>t)$}

The set of ODEs describing the depuration phase $\left(t>t_{c}\right)$ is as follows:

$$
\left\{\begin{array}{l}
\frac{d C_{p}(t)}{d t}=-(E+M) \times C_{p}(t) \\
\frac{d C_{m \ell}(t)}{d t}=k_{m \ell} \times C_{p}(t)-k_{e \ell} \times C_{m \ell}(t), \forall \ell=1 \cdots L
\end{array}\right.
$$

The definitions and units (when applicable) of the parameters and variables are described together in Table 1.

Equation (11.a) is a linear first-order ODE without a second member, so that it has a general solution of the following form:

$$
C_{p}(t)=K \times e^{-(E+M) \times t}, K \in \mathbb{R}^{+}
$$

For the depuration phase and the parent compound, the initial condition comes from the calculation of the internal parent compound concentration at the end of the accumulation phase (i.e., at time $t=t_{\mathrm{c}}$ ) using Equation (13):

$$
C_{p}\left(t_{c}\right)=\left(C_{0}-R\right) \times e^{-(E+M) \times t_{c}}+R
$$

From the general analytical solution given by Equation (12), we get $C_{p}\left(t_{c}\right)=K e^{-(E+M)_{c}}$, from which constant $K$ in Equation (12) is determined by:

$$
K=C_{0}-R+R \times e^{(E+M) \times t_{C}}
$$

Then, the final analytical solution of Equation (11.a), providing the internal concentration of the parent compound over time during the depuration phase $\left(t>t_{c}\right)$, becomes: 


$$
C_{p}(t)=\left(C_{0}-R \times\left(1-e^{(E+M) \times t_{c}}\right)\right) \times e^{-(E+M) \times t}
$$

For simplicity reasons, Equation (15) can be written as $C_{p}(t)=$ $Q e^{-(E+M) \times t}$, where $Q$ is defined in Table 1 .

Equation (11.b) is a linear first-order ODE with constant coefficients and a second member. The analytical solution of Equation (11.b) without its second member is:

$$
C_{m_{\ell}}(t)=K \times e^{-k_{\ell} e_{\ell}}, K \in \mathbb{R}^{+}
$$

As previously stated, the method of the variation of a constant provides the general solution of Equation (11.b) as $C_{m_{\ell}}(t)=K(t) e^{-k_{e} t}$ , from which function $K(t)$ must be determined.

The derivative of $C_{m_{t}}(t)$ from Equation (16) is:

$$
\frac{d K(t)}{d t}=e^{-k_{e_{\ell}} \times t}-k_{e_{\ell}} \times K(t) \times e^{-k_{e_{\ell}} \times t}
$$

Inserting Equation (17) into Equation (11.b) leads to:

$$
\frac{d K(t)}{d t}=k_{m_{\ell}} \times Q \times e^{D_{\ell} \times t}
$$

which integrates into:

$$
K(t)=k_{m_{\ell}} \times \frac{Q}{D_{\ell}} \times e^{D_{\ell} \times t}+C, C \in \mathbb{R}
$$

finally leading to the general analytical solution of Equation (11.b):

$$
C_{m_{\ell}}(t)=k_{m_{\ell}} \times \frac{Q}{D_{\ell}} \times e^{-(E+M) \times t}+C \times e^{-k_{e_{\ell}} \times t}, C \in \mathbb{R}
$$

Constant $C$ can be determined from the initial condition, i.e., from the internal concentration of metabolite $\ell$ at $t=t_{\mathrm{c}}$ both at the end of the accumulation phase and at the beginning of the depuration phase. From Equation (20), we get $C_{m_{e}}\left(t_{c}\right)=k_{m_{e}} \frac{Q}{D} e^{-(E+M)_{c}}+C e^{-k_{e} t_{c}^{c}}$, and from Equation (10), we obtain $C_{m_{\ell}}\left(t_{c}\right)=k_{m_{\ell}}\left(\frac{C_{0}-R}{D_{\ell}}\left(e^{-(E+M) t_{c}}-e^{-k_{e_{\ell}} t_{c}^{c}}\right)+\frac{R}{k_{c_{f}}}\left(1-e^{-k_{c_{e} t_{c}}}\right)\right)$. Finally, the expression for constant $C$ can be determined using:

$$
C=k_{m_{\ell}} \times\left(\frac{R}{k_{e_{\ell}}} \times\left(e^{k_{e_{\ell} \times t_{c}}}-1\right)-\frac{C_{0}-R}{D_{\ell}}-\frac{R}{D_{\ell}} \times e^{k_{e_{\ell}} \times t_{c}}\right)
$$

Replacing constant $C$ in Equation (20) gives the final analytical solution of Equation (11.b), which yields the internal concentration of metabolite $\ell$ for the depuration phase $\left(t>t_{c}\right)$ as follows:

$$
\begin{aligned}
C_{m_{\ell}}(t)= & k_{m_{\ell}} \times\left(\frac{Q}{D_{\ell}} \times e^{-(E+M) \times t}+\frac{R}{k_{e_{\ell}}} \times\left(e^{-k_{e_{\ell}} \times\left(t-t_{c}\right)}-e^{-k_{e_{\ell}} \times t}\right)\right. \\
& \left.-\frac{C_{0}-R}{D_{\ell}} \times e^{-k_{e_{\ell}} \times t}-\frac{R}{D_{\ell}} \times e^{-k_{e_{\ell}} \times\left(t-t_{c}\right)}\right)
\end{aligned}
$$

Replacing constant $Q$ by its own expression (Table 1) leads to:

$$
\begin{aligned}
C_{m_{\ell}}(t)= & k_{m_{\ell}} \times\left(\frac{C_{0}-R}{D_{\ell}} \times\left(e^{-(E+M) \times t}-e^{-k_{e_{\ell}} \times t}\right)\right. \\
& +\frac{R}{k_{e_{\ell}}} \times\left(e^{-k_{e_{\ell}} \times\left(t-t_{c}\right)}-e^{-k_{e_{\ell}} \times t}\right) \\
& +\frac{R}{D_{\ell}} \times\left(e^{-(E+M) \times\left(t-t_{c}\right)}-e^{-k_{e_{\ell}} \times\left(t-t_{c}\right)}\right)
\end{aligned}
$$

\section{Generic set of solutions}

Considering the following intermediate notations $R=\frac{U}{E+M}$ and $D_{\ell}$ $=k_{e_{e}}-(E+M)$, we can obtain the full set of analytical solutions corresponding to the whole one-compartment TK set of ODE describing the time-course for the accumulation and depuration phases of the parent compound and its potential metabolites.

- The analytical solution for the internal concentration of the parent compound during the accumulation phase, previously referred as Equation (3), becomes:

$$
C_{p}(t)=\left(C_{0}-\frac{U}{E+M}\right) \times e^{-(E+M) \times t}+\frac{U}{E+M}, \forall 0 \leqslant t \leqslant t_{c}
$$

- The analytical solution for the internal concentration of metabolite $\ell$ during the accumulation phase, previously referred as Equation (10), is:

$$
\begin{aligned}
C_{m_{\ell}}(t)= & \frac{k_{m_{\ell}}}{k_{e_{\ell}}} \times \frac{U}{E+M} \times\left(1-e^{-k_{e_{\ell}} \times t}\right)+\frac{k_{m_{\ell}}}{k_{e_{\ell}}-(E+M)} \\
& \times\left(C_{0}-\frac{U}{E+M}\right) \times\left(e^{-(E+M) \times t}-e^{-k_{e_{\ell}} \times t}\right), \forall 0 \leqslant t \leqslant t_{c}
\end{aligned}
$$

- The analytical solution for the internal concentration of the parent compound during the depuration phase, previously referred as Equation (15), is written as:

$$
C_{p}(t)=\left(C_{0}-\frac{U}{E+M} \times\left(1-e^{(E+M) \times t_{c}}\right)\right) \times e^{-(E+M) \times t}, \forall t>t_{c}
$$

- The analytical solution for the internal concentration of metabolite $\ell$ during the depuration phase, previously referred as Equation (23), is determined using:

$$
\begin{aligned}
C_{m_{\ell}}(t) & =\frac{k_{m_{\ell}}}{k_{e_{\ell}}-(E+M)} \times\left(C_{0}-\frac{U}{E+M}\right) \times\left(e^{-(E+M) \times t}-e^{-k_{e_{\ell}} \times t}\right) \\
& +\frac{k_{m_{\ell}}}{k_{e_{\ell}}} \times \frac{U}{E+M} \times\left(e^{-k_{e_{\ell}} \times\left(t-t_{c}\right)}-e^{-k_{e_{\ell}} \times t}\right) \\
& +\frac{k_{m_{\ell}}}{k_{e_{\ell}}-(E+M)} \times \frac{U}{E+M} \times\left(e^{-(E+M) \times\left(t-t_{c}\right)}-e^{-k_{e_{\ell}} \times\left(t-t_{c}\right)}\right), \forall t>t_{c}
\end{aligned}
$$

Then, the final generic analytical solution of the one-compartment TK model can be achieved to estimate all parameters from observed data using an inference process by replacing constants $U$, $E$ and $M$ with $U=\sum_{i=1}^{I} k_{u_{i}} \times c_{i}, E=\sum_{j=1}^{J} k_{e_{j}}$ and $M=\sum_{\ell=1}^{L} k_{m_{\ell}}$, respectively.

\section{Model simulations based on real case-studies}

Currently, the above exact mathematical solution of the generic onecompartment TK model is used in support of the development of the R-package rbioacc $^{\mathbf{2 0}}$ as well as its web interface MOSAIC hioacc $_{\text {, }}$ which is freely available at https://mosaic.univ-lyon1.fr/bioacc. ${ }^{21}$ Both tools allow fitting the TK model to bioaccumulation data in a user-friendly way, providing all necessary outputs to check for goodness-of-fit and obtain bioaccumulation metrics, regardless of the considered speciescompound combination. The database associated to the web interface is also available online at http://lbbe-shiny.univ-lyon 1.fr/mosaicbioacc/data/database/TK_database.html, with a wide collection of accumulation-depuration data in support of toxicokinetic modelling. ${ }^{22}$ 
Table 2. Sets of inputs for model simulations according to Figures 1 to 5

\begin{tabular}{|c|c|c|c|c|}
\hline Input & Units & Simple (section 4.1) & Multiple exposures (section 4.2) & Biotransformation (section 4.3) \\
\hline$c_{w}$ & $\mu g \cdot m L^{-1}$ & 0.0044 & - & 15.53 \\
\hline$c_{s}$ & $\mu g \cdot g^{-1}$ & - & 56.6 & - \\
\hline$c_{f}$ & $\mu g \cdot g^{-1}$ & - & 1.46 & - \\
\hline$t_{c}$ & days & 49 & 7 & 1 \\
\hline$k_{u_{w}}$ & days $^{-1}$ & 10.46 & - & 16,740 \\
\hline$k_{u_{s}}$ & days $^{-1}$ & - & 0.071 & - \\
\hline$k_{u_{f}}$ & days $^{-1}$ & - & 0.013 & - \\
\hline$k_{m_{1}}$ & days $^{-1}$ & - & - & 73.27 \\
\hline$k_{m_{2}}$ & days $^{-1}$ & - & - & 0.5166 \\
\hline$k_{m_{3}}$ & days $^{-1}$ & - & - & 0.1957 \\
\hline$k_{e_{e}}$ & days $^{-1}$ & 0.04 & 0.178 & 4.164 \\
\hline$k_{e_{1}}$ & days $^{-1}$ & - & - & 561 \\
\hline$k_{e_{2}}$ & days $^{-1}$ & - & - & 0.123 \\
\hline$k_{e_{3}}$ & days $^{-1}$ & - & - & 0.7808 \\
\hline
\end{tabular}

(-) Hyphens stand for no required inputs.

From this database, we selected three real case-studies from which we got fitting results (especially the joint posterior probability distribution of all kinetic parameters) in order to perform the subsequent model simulations, thus illustrating the use of the generic TK model.

All model simulations of the generic set of mathematical solutions (Equations (3), (10), (15) and (23)) were performed using R software with the rbioacc package and function predict (). ${ }^{20}$ Note that the same simulations can be performed directly online with the $\mathrm{MOSAIC}_{\text {bioacc }}$ platform and its prediction module (https://mosaic.univ-lyon1.fr/bioacc). We used 500 time points in $\left[0 ; t_{f}\right]$, where $t_{f}$ stands for the final time of each simulation. These simulations illustrate the use of the generic TK model in three case studies where different compounds are bioaccumulated by different species. In order to proceed, the time duration of the accumulation phase (parameter $t_{c}$ ) is required, as well as the exposure concentrations in the media and the model parameter values (Table 2); all associated numerical values were taken from fitting results performed with real data sets. Figures 1 to 5 show the simulations of internal concentrations over time for the three speciescompound combinations, as subsequently described. For each case study, TK model parameters were varied one-at-a-time with 20, 50, and $80 \%$ of increase from their original value.

\section{Elementary one-compartment TK model}

Equations (3) and (15) were simulated for a simple case study in which fish are exposed to a highly hydrophobic chemical contaminated water and only excretion is considered (Fig. 1 and Table 2). ${ }^{5}$ The corresponding inputs are the exposure concentration $c_{w}$ (referring to $c_{i}$ and $I=1$ ), uptake rate from water $k_{u_{w}}$ (referring to $k_{u_{i}}$ and $I=1)$, and excretion rate $k_{e_{e}}$ (referring to $k_{e_{j}}$ and $\left.J=1\right)$. When parameter $k_{u_{w}}$ increases, internal concentrations become higher (e.g., blue curve in Fig. 1a) than with the original value (orange curve in Fig. 1a). Biologically speaking, the higher the uptake rate is for a given substance, the more it is bioaccumulated by organisms. Conversely, an increase in parameter $k_{e}$ leads to a lower internal concentration (e.g., blue curve in Fig. 1b), which is consistent with the known underlying biological mechanism: the faster a contaminant is eliminated, the quicker its concentration decreases in organisms.

\section{One-compartment TK model with several exposure routes, no metabolites}

Equations (3) and (15) are simulated for case, in which freshwater shrimp are exposed to an organic chlorine compound through contaminated sediment and food, and only natural excretion is considered as the elimination process (Fig. 2 and Table 2). ${ }^{23}$ The corresponding inputs are the exposure concentration via sediment $c_{s}$ and food $c_{f}$ (referring to $c_{i}$ and $I=2$ ), the two uptake rates from sediment and food, $k_{u_{s}}$ and $k_{u_{f}}$ (referring to $k_{u_{i}}$ and $I=2$ ), and excretion rate $k_{e_{e}}\left(\right.$ referring to $k_{e_{j}}$ and $\left.J=1\right)$. When parameter $k_{u_{s}}$ increases, 

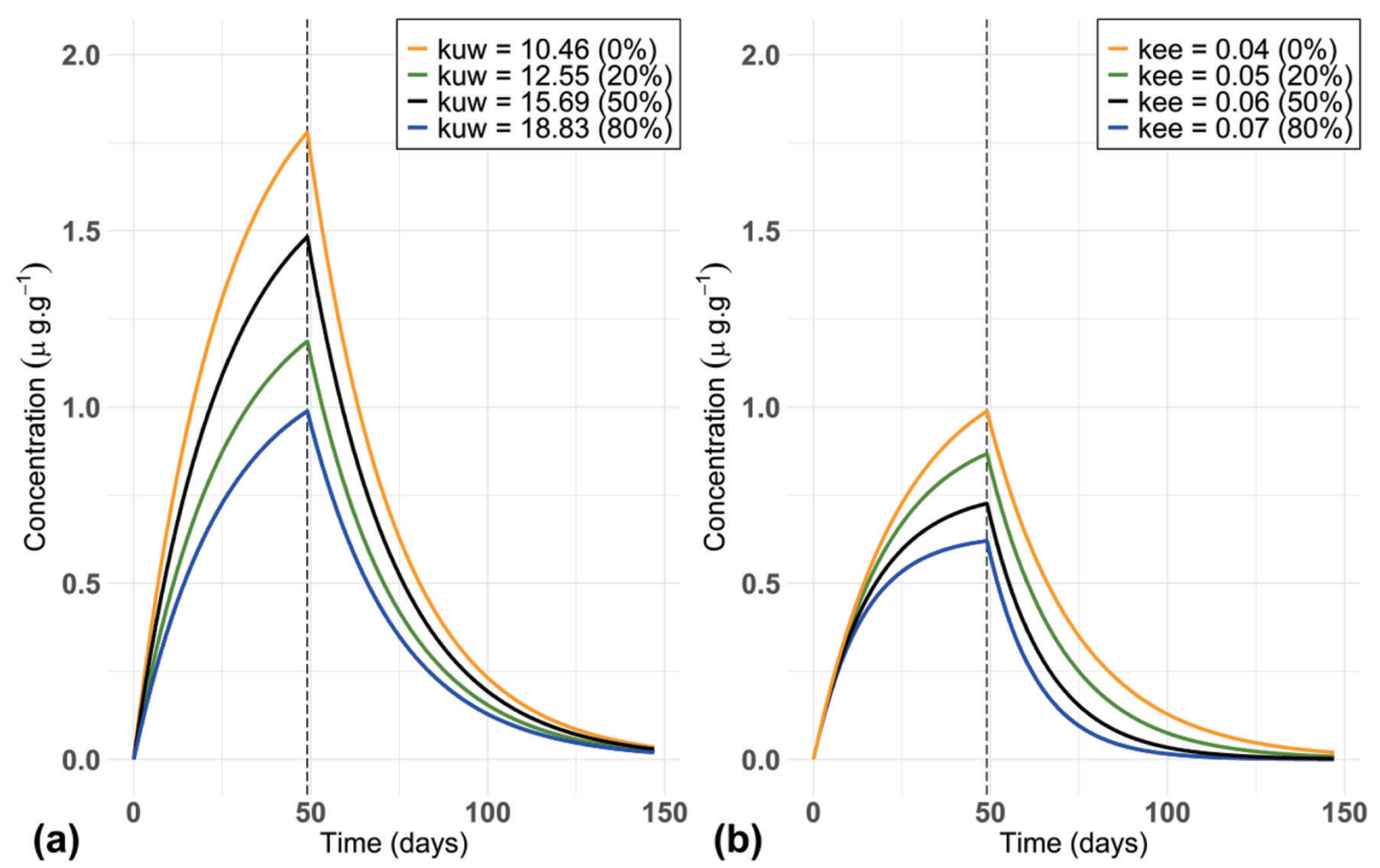

Fig. 1. Example of model simulations for a simple TK model (exposure via water and elimination by excretion) and the influence of variations of parameters (a) $k_{u_{w}}$ (uptake rate) and (b) $k_{e_{e}}$ (excretion rate). TK, toxicokinetic.

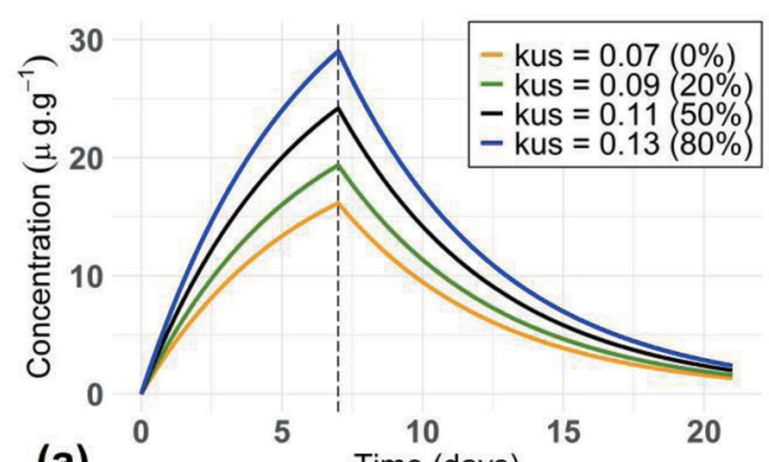

(a)

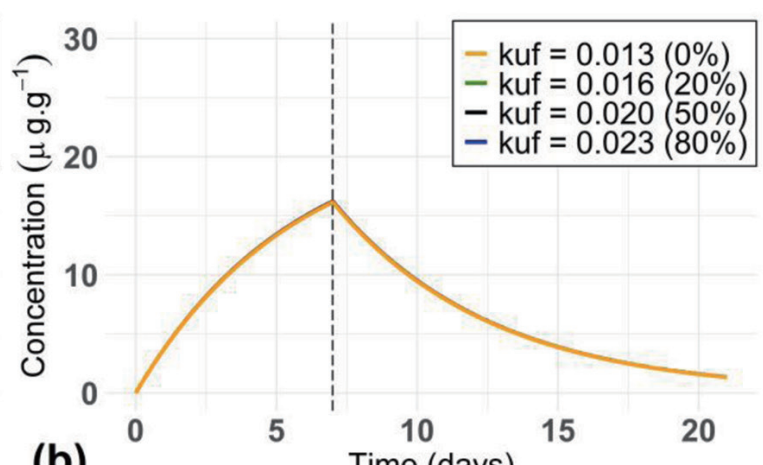

(b) Time (days)

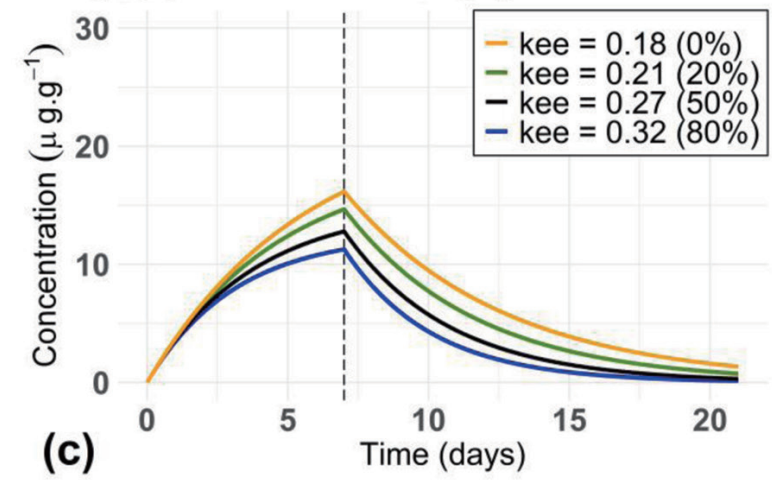

Fig. 2. Example of model simulations for a TK model with multiple exposure routes (via sediment and food) and influence of variations of parameters (a) $\boldsymbol{k}_{u_{s}}$ (uptake rate from sediment), (b) $\boldsymbol{k}_{u_{f}}$ (uptake rate from food) and (c) $\boldsymbol{k}_{e_{e}}$ (excretion rate). TK, toxicokinetic. 

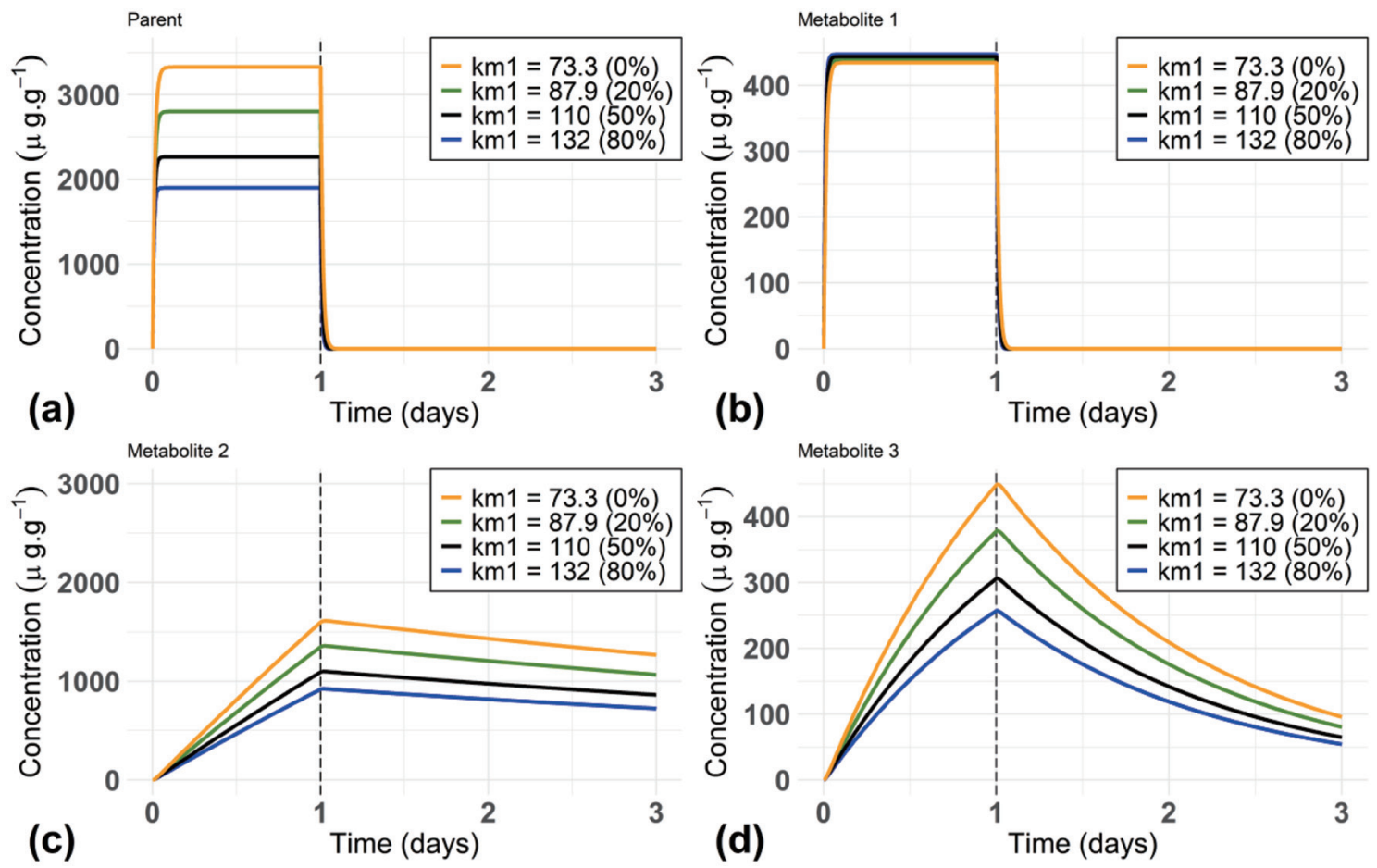

Fig. 3. Example of model simulations for a TK model with biotransformation (three metabolites) and influence of the variations of parameter $k_{m}$ (biotransformation rate for metabolite 1 ) on (a) the parent compound, (b) metabolite 1, (c) metabolite 2, and (d) metabolite 3 . TK, toxicokinetic.

internal concentrations are higher (e.g., blue curve in Fig. 2a) than with the original value (orange curve in Fig. 2a). Biologically, the higher the uptake rate from sediment is for a given substance, the stronger it is bioaccumulated by organisms. Regarding the exposure from food, as parameter $k_{u f}$ is low in this case, its variation has minimal influence on the internal concentration of the contaminant (Fig. 2b). This means that the exposure via sediment is the major route of contamination for these organisms. Besides, as previously shown in section 4.1 , an increase in parameter $k_{e}$ leads to a faster decreasing concentration (e.g., blue curve in Fig. $2 c)$.

\section{One-compartment TK model with one exposure route, several metabolites}

Equations (3), (10), (15) and (23) are simulated for case, in which freshwater shrimp are exposed to an organic biocide by contaminated water. Three metabolites derived from the parent compound are considered ${ }^{24}$ together with the natural excretion (Figs. 3 to 5 and Table 2). The corresponding inputs are the exposure concentration via water $c_{w}$ (referring to $c_{i}$ and $\left.I=1\right)$, the uptake rate $k_{u_{w}}$ (referring to $k_{u_{i}}$ and $I=1$ ), excretion rate $k_{e_{e}}$ (referring to $k_{e_{j}}$ and ${ }^{u_{w}} J$ $=1$ ), three metabolization rates $k_{m_{1}}, k_{m_{2}}$ and $k_{m_{3}}$ (referring to $k_{m_{\ell}}$ and $L=3$ ), and three elimination rates of the metabolites $k_{e_{1}}, k_{e_{2}}$ and $k_{e_{3}}$ (referring to to $k_{e_{\ell}}$ and $L=3$ ). When parameter $k_{m_{1}}$ increases, internal concentrations of metabolite 1 increase (e.g., blue curve in Fig. 3b) greater than with the original value (orange curve in Fig. 3b). Conversely, internal concentrations are lower for the parent compound (e.g., blue curve in Fig. 3a). In other words, the more the biotransformation rate for a given metabolite increases, the higher its concentration becomes within organisms due to the highly biotransformation of the parent compound. This leads to a lower internal concentration of the parent compound than with the original value of $k_{m_{1}}$ (e.g., blue curve, Fig. 3a). An increase in $k_{m_{1}}$ also induces a decrease in the internal concentrations of the other metabolites (Figs. $3 \mathrm{c}$ and d). Besides, an increase in parameter $k_{e_{1}}$ only affects the internal concentration of metabolite 1 (Fig. 4b). In addition, as previously viewed (sections 4.1 and 4.2), an increase in parameter $k_{u_{w}}$ will induce high internal concentrations for both the parent compound and its metabolites (Fig. 5). Indeed, the more the internal concentration of the parent compound increases, the more the biotransformation process will intensify, leading to high internal concentrations for each metabolite.

\section{Future directions}

Based on the results of this work, we suggest future research aims to acquire a better understanding of the variation of TK parameters according to the chemicals and their exposure concentrations in the environment. In particular, the influence of concentration dependency may be investigated through the TK parameter values. For example, if the uptake rate remains on the same order of magnitude rather than increasing two-fold when the exposure concentration doubles, this would most likely indicate a concentration dependency. A second interesting research direction would be the elaboration of an improved modelling framework that includes phase II metabolites within the TK model; some experimental data are already available to support such research but no model exists yet.

\section{Conclusions}

As supported by complete mathematical and exact calculations, our work introduces a practical and generic one-compartment TK 

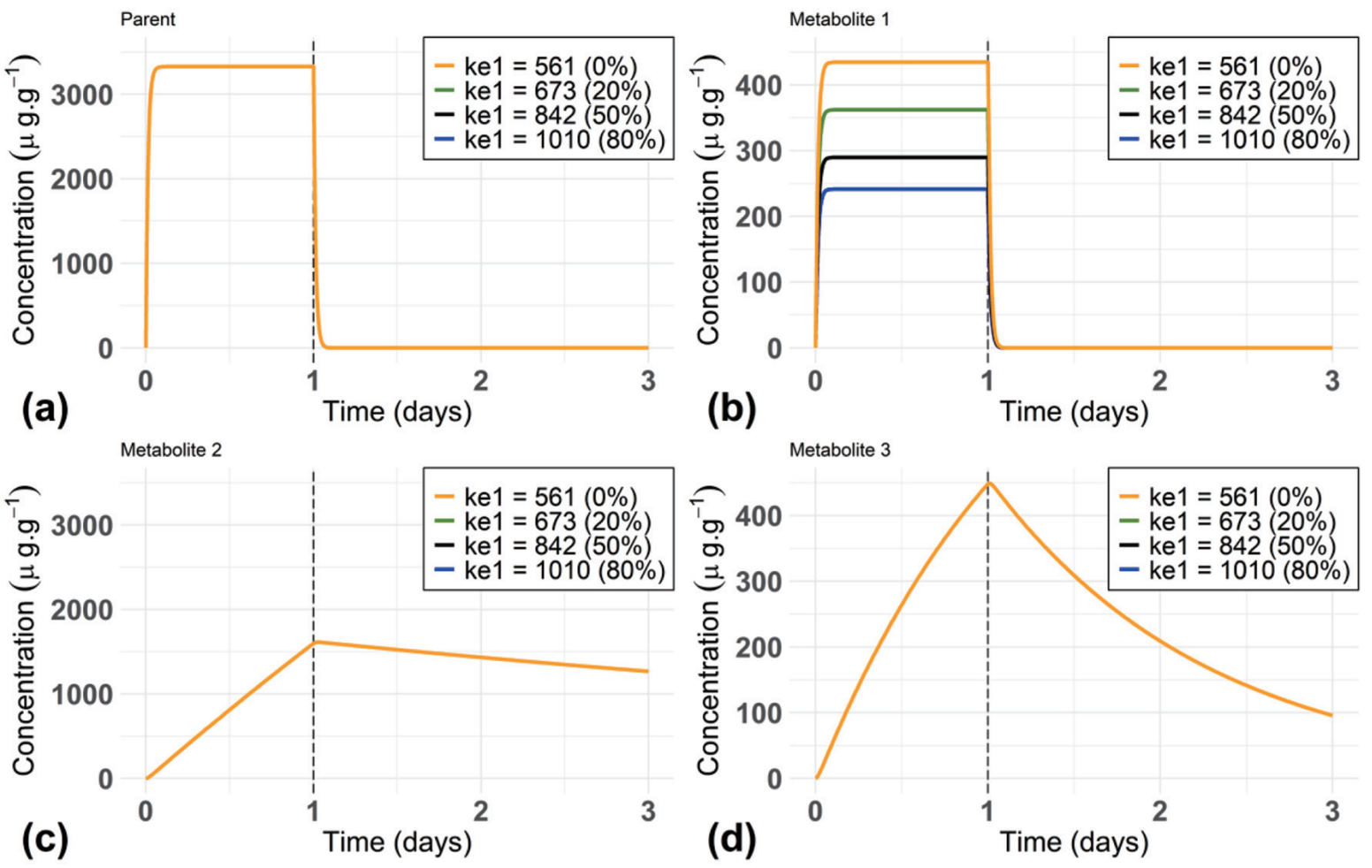

Fig. 4. Example of model simulations for a TK model with biotransformation (three metabolites) and influence of the variations of parameter $k_{e_{1}}$ (elimination rate of metabolite 1) on (a) the parent compound, (b) metabolite 1, (c) metabolite 2, and (d) metabolite 3. TK, toxicokinetic.
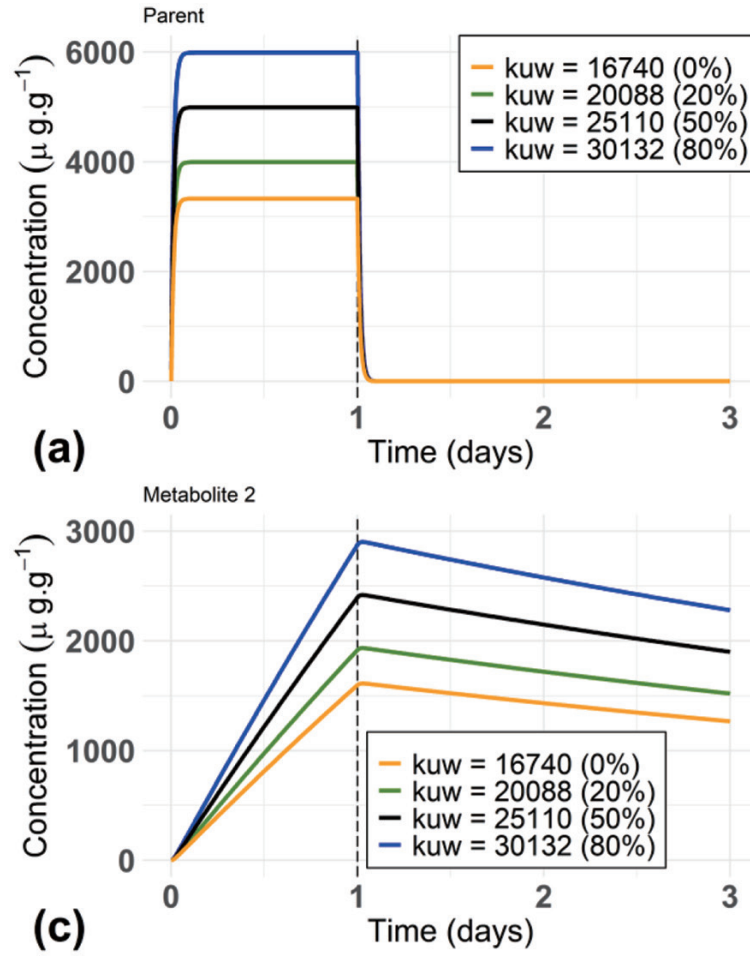

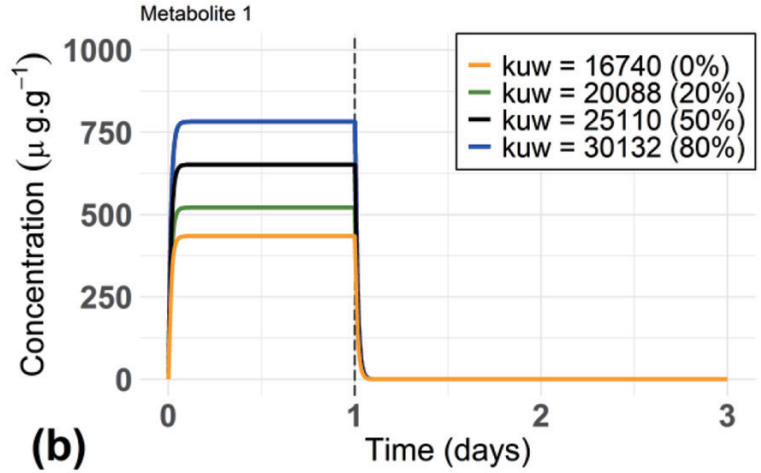

Metabolite 3

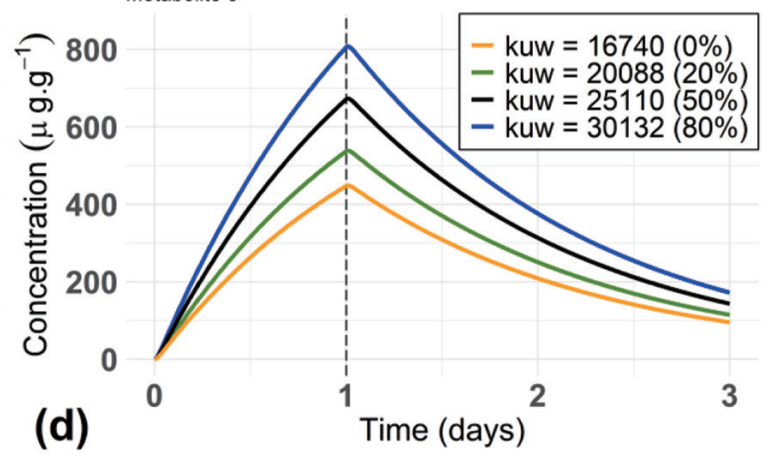
Fig. 5. Example of model simulations for a TK model with biotransformation (three metabolites) and influence of the variations of parameter $\boldsymbol{k}_{u_{w}}$ on (a)
the parent compound, (b) metabolite 1, (c) metabolite 2, and (d) metabolite 3. TK, toxicokinetic. 
model to deal with different species-compound combinations of interest. To-date, such a model is required by regulatory bodies to perform risk assessment of chemical substances. The generic feature of this model is, thus, in full compliance with the numerous situations that regulators are expected to be faced. The associated ready-to-use tools we developed to perform different actions, including calibration of experimental data, validation of simulations compared to observed data, and predictions in untested situations, should be of pertinent use to such regulators.

\section{Acknowledgments}

The authors would like to express their sincere thanks to Miléna Kaag, Yacout Lahlou and Nino Molin who designed the MOSAI$\mathrm{C}_{\text {bioacc }}$ prediction tool as part of their $4^{\text {th }}$ year study project at the National Institute of Applied Sciences (INSA) in Lyon (France). This work was made under the umbrella of the French GDR "Aquatic Ecotoxicology" framework, aiming at fostering stimulating scientific discussions and collaborations for more integrative approaches.

\section{Funding}

This work was financially supported by the Graduate School H2O'Lyon (ANR-17-EURE-0018) and "Université de Lyon" (UdL), as part of the program "Investissements d'Avenir" run by "Agence Nationale de la Recherche" (ANR).

\section{Conflict of interest}

The authors have no conflicts of interest related to this publication.

\section{Author contributions}

Contributed to study concept and design (SC, AR and CL), generic solving (SC, CL), acquisition of the data (AR), assay model simulations (AR), drafting of the manuscript ( $\mathrm{SC}, \mathrm{AR}$ and $\mathrm{CL}$ ), supervision (SC, CL).

\section{Data sharing statement}

No additional data are available.

\section{References}

[1] Gupta P. General Principles of Toxicology. Concepts and Applications in Veterinary Toxicology. Springer, Cham; 2019. doi:10.1007/978-3-03022250-5_1.

[2] Côté J, Bonvalot Y, Carrier G, Lapointe C, Fuhr U, Tomalik-Scharte D, et al. A novel toxicokinetic modeling of cypermethrin and permethrin and their metabolites in humans for dose reconstruction from biomarker data. PLoS One 2014;9(2):e88517. doi:10.1371/journal. pone. 0088517.

[3] Paini A, Worth A, Kulkarni S, Ebbrell D, Madden J. Assessment of the predictive capacity of a physiologically based kinetic model using a read-across approach. Comput Toxicol 2021;18:100159. doi:10.1016/j. comtox.2021.100159.
[4] Lautz LS, Hoeks S, Oldenkamp R, Hendriks AJ, Dorne JLCM, Ragas AMJ. Generic physiologically based kinetic modelling for farm animals: Part II. Predicting tissue concentrations of chemicals in swine, cattle, and sheep. Toxicol Lett 2020;318:50-56. doi:10.1016/j.toxlet.2019.10.008.

[5] Lautz LS, Dorne JLCM, Oldenkamp R, Hendriks AJ, Ragas AMJ. Generic physiologically based kinetic modelling for farm animals: Part I. Data collection of physiological parameters in swine, cattle and sheep. Toxicol Lett 2020;319:95-101. doi:10.1016/j.toxlet.2019.10.021.

[6] Riviere JE, Papich MG, editors. Veterinary Pharmacology and Therapeutics. Wiley-Blackwell, 2017. Available from: https://www.wiley. com/en-us/Veterinary+Pharmacology+and+Therapeutics\%2C+10th+E dition-p-9781118855881 Accessed September 8, 2021.

[7] Gehring R. Veterinary-specific applications of pharmacokinetic modelling and theory: Opportunities and challenges [Dissertation]. Nijmegen: Radboud University; 2020.

[8] Molnar J. Physiologically Based Pharmacokinetic Modeling-Science and Applications. Am J Ther 2006;13(2):177.

[9] McLanahan ED, El-Masri HA, Sweeney LM, Kopylev LY, Clewell HJ, Wambaugh JF, et al. Physiologically based pharmacokinetic model use in risk assessment-Why being published is not enough. Toxicol Sci 2012;126(1):5-15. doi:10.1093/toxsci/kfr295.

[10] Organisation for Economic Cooperation and Development (OECD). Test No. 305: Bioaccumulation in Fish: Aqueous and Dietary Exposure. OECD Guidelines for the Testing of Chemicals, Section 3. Paris: OECD Publishing; 2012. doi:10.1787/9789264185296-en.

[11] Nyman AM, Schirmer K, Ashauer R. Importance of toxicokinetics for interspecies variation in sensitivity to chemicals. Environ Sci Technol 2014;48(10):5946-5954. doi:10.1021/es5005126.

[12] D'Argenio DZ, Bae KS. Analytical solution of linear multi-compartment models with non-zero initial condition and its implementation with R. Transl Clin Pharmacol 2019;27(2):43-51. doi:10.12793/ tcp.2019.27.2.43.

[13] Valcke M, Bourgault M H, Gagné M, Levallois P. A probabilistic toxicokinetic modeling approach to the assessment of the impact of daily variations of lead concentrations in tap water from schools and daycares on blood lead levels in children. Sci Total Environ 2021;775:145866. doi:10.1016/j.scitotenv.2021.145866.

[14] Sasso AF, Isukapalli SS, Georgopoulos PG. A generalized physiologicallybased toxicokinetic modeling system for chemical mixtures containing metals. Theor Biol Med Model 2010;7(1):1-17. doi:10.1186/17424682-7-17.

[15] Grech A, Brochot C, Dorne JL, Quignot N, Bois FY, Beaudouin R. Toxicokinetic models and related tools in environmental risk assessment of chemicals. Sci Total Environ 2017;578:1-15. doi:10.1016/j.scitotenv.2016.10.146.

[16] Mavroudis PD, Hermes HE, Teutonico D, Preuss TG, Schneckener S. Development and validation of a physiology-based model for the prediction of pharmacokinetics/toxicokinetics in rabbits. PLoS One 2018;13(3):e0194294. doi:10.1371/journal.pone.0194294.

[17] Gestin O, Lacoue-Labarthe T, Coquery M, Delorme N, Garnero L, Dherret $\mathrm{L}$, et al. One and multi-compartments toxico-kinetic modeling to understand metals' organotropism and fate in Gammarus fossarum. Environ Int 2021;156:106625. doi:10.1016/j.envint.2021.106625.

[18] Ratier A, Lopes C, Labadie P, Budzinski H, Delorme N, Quéau H, et al. A Bayesian framework for estimating parameters of a generic toxicokinetic model for the bioaccumulation of organic chemicals by benthic invertebrates: Proof of concept with PCB153 and two freshwater species. Ecotoxicol Environ Saf 2019;180:33-42. doi:10.1016/j. ecoenv.2019.04.080.

[19] Kuo DT, Chen CC. Deriving in vivo biotransformation rate constants and metabolite parent concentration factor/stable metabolite factor from bioaccumulation and bioconcentration experiments: An illustration with worm accumulation data. Environ Toxicol Chem 2016;35(12):2903-2909. doi:10.1002/etc.3509.

[20] Ratier A, Baudrot V, Kaag M, Siberchicot A, Lopes C, Charles S. rbioacc: an R-package to analyse toxicokinetic data. bioRxiv [Preprint] 2021;2021.09.08.459421. doi:10.1101/2021.09.08.459421.

[21] Ratier A, Lopes C, Multari G, Mazerolles V, Carpentier P, Charles S. New perspectives on the calculation of bioaccumulation metrics for active substances in living organisms. Integr Environ Assess Manag 2021;doi:10.1002/ieam.4439. 
[22] Ratier A, Charles S. Accumulation-depuration data collection in support of toxicokinetic modelling. bioRxiv [Preprint] 2021;2021.04.15.439942. doi:10.1101/2021.04.15.439942.

[23] Crookes MJ, Brooke DN. Estimation of fish bioconcentration factor (BCF) from depuration data. Bristol: Environment Agency, Horizon House; 2011. Available from: https://www.gov.uk/government/pub- lications/estimation-of-fish-bioconcentration-factor-bcf-from-depuration-data Accessed September 8, 2021.

[24] Ashauer R, Hintermeister A, O'Connor I, Elumelu M, Hollender J, Escher BI. Significance of xenobiotic metabolism for bioaccumulation kinetics of organic chemicals in Gammarus pulex. Environ Sci Technol 2012;46(6):3498-508. doi:10.1021/es204611h. 